\title{
Network Coding with Multimedia Transmission and Cognitive Networking: An Implementation based on Software-Defined Radio
}

\author{
Tran Thi Thuy Quynh ${ }^{1}$, Ngo Khac Hoang ${ }^{1,2}$, Nguyen Van Ly,3, Nguyen Linh Trung ${ }^{1}$, \\ Nguyen Quoc Tuan ${ }^{1}$, Ejder Bastug ${ }^{4}$, Sylvain Azarian ${ }^{5}$, Le Vu Ha ${ }^{1}$, Vo Nguyen Quoc Bao ${ }^{6}$, \\ Tran Xuan Nam ${ }^{7}$, Mérouane Debbah ${ }^{8}$, Pierre Duhamel ${ }^{9}$ \\ ${ }^{1}$ University of Engineering and Technology, Vietnam National University, Hanoi, Vietnam \\ 2 Department of Electrical Engineering, Chalmers University of Technology, 41296 Gothenburg, Sweden \\ ${ }^{3}$ Computational Science Research Center, San Diego State University, CA, USA \\ ${ }^{4}$ Nokia Bell Labs, 91620 Nozay, France \\ 5 SDR-Technologies Ltd., France \\ ${ }^{6}$ Posts and Telecommunications Institute of Technology, Ho Chi Minh City, Vietnam \\ ${ }^{7}$ Faculty of Radio Electronics, Le Quy Don Technical University, Hanoi, Vietnam \\ ${ }^{8}$ Huawei Lagrange Mathematical \& Computing Research Center, 75007 Paris, France \\ 9 Signals and Systems Laboratory, CentraleSupelec, Paris-Saclay University, 91190 Gif-sur-Yvette, France
}

Correspondence: Nguyen Linh Trung, linhtrung@vnu.edu.vn

Communication: received 29 December 2020, revised 31 December 2020, accepted 31 December 2020

Online publication: 25 February 2021, Digital Object Identifier: 10.21553/rev-jec.263

The associate editor coordinating the review of this article and recommending it for publication was Prof. Nguyen Tan Hung.

\begin{abstract}
Network coding (NC) is considered a breakthrough to improve throughput, robustness, and security of wireless networks. Although the theoretical aspects of NC have been extensively investigated, there have been only few experiments with pure NC schematics. This paper presents an implementation of NC under a two-way relay model and extends it to two non-straightforward scenarios: (i) multimedia transmission with layered coding and multiple-description coding, and (ii) cognitive radio with Vandermonde frequency division multiplexing (VFDM). The implementation is in real time and based on software-defined radio (SDR). The experimental results show that, by combining NC and source coding, we can control the quality of the received multimedia content in an on-demand manner. Whereas in the VFDM-based cognitive radio, the quality of the received content in the primary receiver is low (due to imperfect channel estimation) yet retrievable. Our implementation results serve as a proof for the practicability of network coding in relevant applications.
\end{abstract}

Keywords- Network coding, two-way relay model, software-defined radio (SDR), orthogonal frequency division multiplexing (OFDM), Vandermonde frequency division multiplexing (VFDM), multimedia, cognitive radio.

\section{INTRODUCTION}

In 2000, network coding (NC) was first introduced by Ahlswede in [1] to improve network throughput. Unlike the store-and-forward mechanism in traditional routing protocols, NC allows intermediate nodes between source and destination to perform additional computations (coding) on the incoming data before forwarding the coded information.

There are two main NC schemes, namely straightforward network coding (SNC) where coding is performed on digital bit streams after they have been received [2], and physical-layer network coding (PNC) where coding is performed via the additive nature of simultaneously arriving electromagnetic waves [3,4]. The latter was shown to achieve a higher throughput performance as illustrated in the following example.

Let us consider a two-way relay (TWR) model depicted in Figure 1(a), which is a simple and popular network topology. In this model, two end nodes $A$ and $B$ expect to exchange their respective data packet $a$ and $b$ with each other via a relay node $R$. It is assumed that there is no direct link between $A$ and $B$ due to, e.g., blockage and limited radio range. We consider three communication schemes.

First, following a traditional store-and-forward scheme, the network needs four time slots to complete the packet exchange, as illustrated in Figure 1(b). Specifically, $A$ transmits packet $a$ to $R$ in the first time slot, and $R$ forwards $a$ to $B$ in the second time slot. Then, $B$ transmits packet $b$ to $R$ in the third time slot, and $R$ forwards $b$ to $A$ in the fourth time slot. Note that node $R$ merely forward the packets without processing the data contents.

Second, following SNC, as illustrated in Figure 1(c), the number of time slots can be reduced. The relay node $R$ first receives sequentially both packets $a$ and $b$, then performs the bit-wise exclusive OR (XOR) operation, 


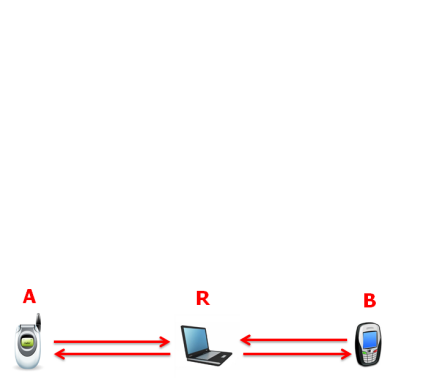

(a) TWR model

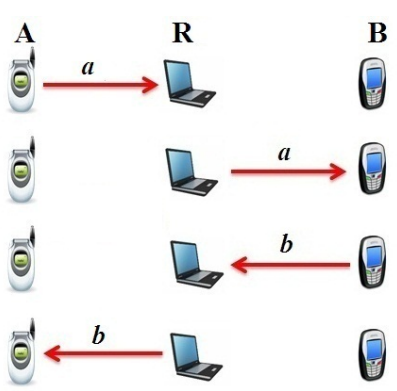

(b) Conventional: 4 time slots

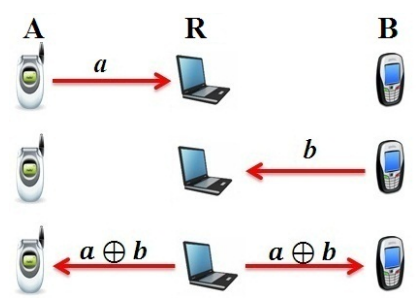

(c) SNC: 3 time slots

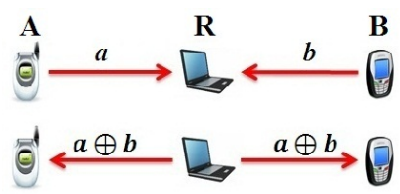

(d) PNC: 2 time slots

Figure 1. Conventional forwarding and network coding methods in the TWR model.

denoted by $\oplus$, over these packets to produce a new single packet $a \oplus b$. In the third time slot, node $R$ simply broadcasts this coded packet. The two end nodes can recover their expected packet based on their own packet and the received coded packet. Specifically, $A$ can recover $b$ as $b=a \oplus(a \oplus b)$ and $B$ can recover $a$ as $a=b \oplus(a \oplus b)$. In this way, SNC reduces the number of time slots to three, achieving a 33\% throughput gain.

Finally, following PNC, illustrated in Figure 1(d), $A$ and $B$ transmit simultaneously. Their packets are combined on the air due to the additive nature of the electromagnetic waves. Node $R$ properly processes the received signal in order to produce the coded packet before forwarding in the second time slot. Therefore, PNC requires only two time slots, achieving a 50\% throughput gain compared to the traditional store-andforward routing scheme.

Although NC has been widely analyzed and assessed via both theoretical analyses and numerical simulations, limited results have been obtained via realchannel implementation. One of the first implementations of NC was reported in [5], where a simplified version of PNC, called analog network coding (ANC), was introduced.

The idea of ANC is that the relay node simply amplifies then retransmits the received superimposed signals without coding. This implementation was based on a software-defined radio (SDR) platform consisting of the Universal Software Radio Peripheral (USRP) with RFX2400 daughterboards as the hardware and the open source GNU Radio [6] as the signal processing software.

Although ANC is simple to implement, it has a critical drawback of error propagation since the relay amplifies the noise along with the signal before forwarding. PNC systems in which the relay performs XOR or other denoising PNC mappings on the re- ceived signals are preferred since they yield significant performance improvements.

The first successful implementation of PNC was reported in [7]. However, this system operates in an offline manner. The first real-time PNC implementation was introduced in [8], based on USRP N210 with XCVR2450 boards. In this system, frequency division duplex (FDD) was employed to separate the uplink and the downlink. To overcome some implementation challenges such as synchronization, packet detection, channel estimation, and carrier-frequency-offset (CFO) estimation, the authors used orthogonal frequency division multiplexing (OFDM) with beacons. In addition, PHY-layer forward error control and MAC-layer ARQ error control were also used to improve reliability. However, this implementation requires a change in the frame format and a balance between the power of the end nodes' signals received at the relay.

Another implementation of NC was presented in [9]. This prototype is for SNC and half-duplex packet switching, also based on USRP with RFX2400 daughterboards. Some other recent implementations of network coding can be found in, for example, [10, 11].

In this paper, we present an SDR-based implementation of NC under the TWR model and two extended network models for multimedia transmission and cognitive radio, respectively. Our main contributions are three-fold. First, we implement NC under the TWR model operating in full-duplex transmission mode. In our scheme, we let node $A$ and node $B$ transmit in separated frequencies in the first time slot, and the relay node performs the XOR operation on digital bit streams and then broadcasts the XOR-ed packet in the second time slot. Compared to existing implementations of NC under the TWR model, our scheme combines the advantages of both SNC and PNC; that is, it requires only two time slots while avoiding complex PNC mapping. Furthermore, the proposed scheme can be easily integrated into existing systems since it does not require any change in the frame format. We also implement an error correction code to improve the reliability. Second, we present the first implementation of NC for multimedia transmission with joint source-network coding. Two types of source coding are considered: layered coding and multiple-description coding. NC is employed to ensure that the destination can still receive data packets when the direct link is lost. Finally, we present the first implementation of NC for a special cognitive radio network based on Vandermonde frequency division multiplexing (VFDM). Specifically, we consider a TWR model in the secondary tier of an overlay two-tier network. To avoid cross-tier interference to the primary receiver, the relay node uses VFDM to transmit signals on the nulls of the primary channel.

The rest of the paper is organized as follows. Section 2 describes our NC framework and prototype, as well as three scenarios whose implementations are subsequently presented. Specifically, Section 3 presents the implementation of NC under the TWR model. Section 4 extends the implementation of $\mathrm{NC}$, combining source coding with multimedia transmission. Section 5 
presents the implementation of NC in the VFDM-based cognitive radio network. Finally, Section 6 gives concluding remarks.

\section{Proposed Network Coding Framework AND PROTOTYPe}

\subsection{Proposed Network Coding Framework}

Following the principle of $\mathrm{NC}$, we present a framework in which relay nodes are inserted in the network to detect and exploit coding opportunities to forward multiple packets in a single transmission in order to improve network throughput and reliability. Consider a general mesh network of which each node has packets to transmit and would like to receive packets from certain other nodes. Our considered framework incorporates the following features.

Relaying infrastructure: Relay nodes are deployed in the network to help forward the packets. These nodes are placed at positions where there is no direct link between the communicating nodes due to blockage or limited radio range, or specially designed so as to maximize the coding gain.

Opportunistic listening: Exploiting the broadcast nature of wireless media, the relay nodes opportunistically listen to the packets transmitted from surrounding nodes. It is assumed that each node includes its identity and the identities of the other nodes from which it would like to receive packets. Thus, the relay nodes can detect this information from the packets.

Opportunistic coding: From knowledge of the desired packets of each node, each relay node identifies the opportunity to encode a subset of the received packets to form a coded packet and broadcast this coded packet. Since there can be multiple coding options corresponding to multiple subsets of the received packets, the coding operation should be well designed. On one hand, the relay nodes should maximize the number of single packets encoded to reduce the transmission time. On the other hand, these coded packets should enable the intended nodes to decode their desired packets. For example, under the TWR model introduced in Section 1, after the first phase, the relay has received packets $a$ and $b$ from nodes $A$ and $B$, respectively. It further knows that node $A$ has packet $a$ while desiring packet $b$, and that node $B$ has packet $b$ while desiring packet $a$. Therefore, a coding opportunity occurs, and the optimal coding operation is to combine $a$ and $b$ and send this combination, $a \oplus b$. In general, a subset of received packets should be combined if all the intended nodes already have all packets in the subset but one packet that they desire. Note that the number of encoded single packets, so-called the coding field size, can be larger than 2 . The field size goes up linearly with the number of nodes in the network.

\subsection{Proposed Prototype}

Within the described framework, we design a prototype with the following elements. First, the nodes transmit their packets using OFDM. Second, in order for the

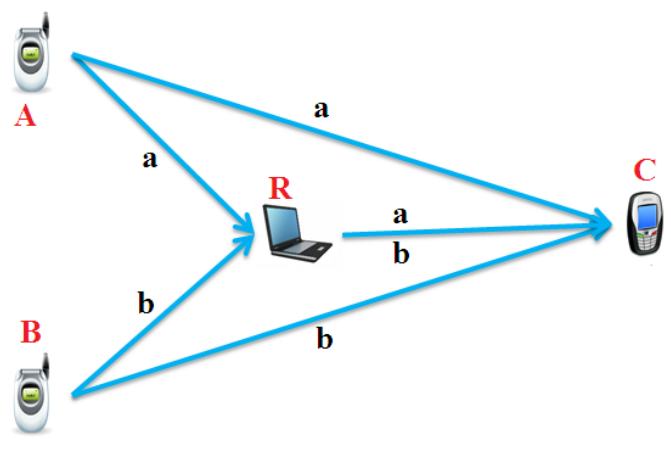

(a) A 4-node network model

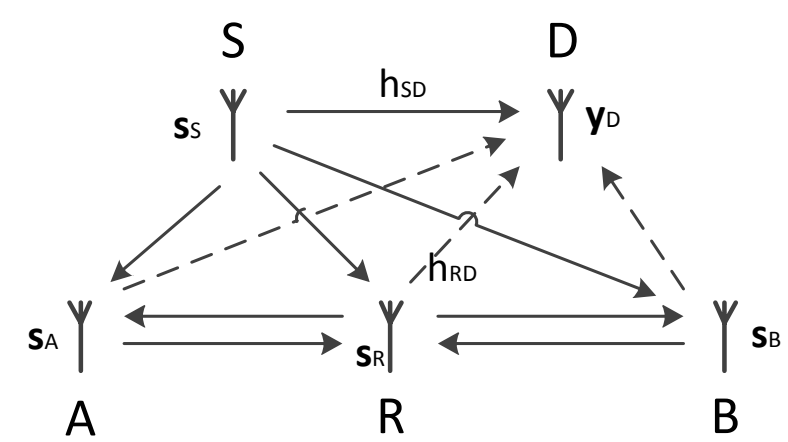

(b) A 2-tier cognitive radio network

Figure 2. Two scenarios of interest for NC implementation, apart from the TWR model.

relay nodes to simultaneously receive the packets, the communicating nodes employ FDD. Specifically, they shift their carrier frequency by a small amount from a central frequency using a frequency mixer, so that their signals are separated. The relay nodes receive at the central frequency and sample with a sufficiently wide bandwidth so as to recover the packets of multiple nodes. Third, we use an SDR platform that consists of bladeRF hardware [12] and GNU Radio software. Each node is a commodity personal computer running GNU Radio and connected to one or more bladeRFx115 devices. The bladeRF devices operate in the fullduplex mode with two associated VERT 2450 antennas for transmission and reception.

We consider three scenarios of NC for implementation as follows. The first scenario is the TWR model presented in Section 1, where nodes $A$ and $B$ would like to exchange packets with the help of a relay. The second scenario, shown in Figure 2(a), is a 4-node network where nodes $A$ and $B$ would like to send packets to a destination node, $C$, with the help of a relay. We assume that the packets from nodes $A$ and $B$ are generated from a specific type of source coding for multimedia transmission. The third scenario, shown in Figure 2(b), is a two-tier cognitive radio network with a transceiver pair at the primary tier and a TWR model at the secondary tier. In these scenarios, we restrict the NC operation to a field size of 2, i.e., the relay node combine the incoming packets from two nodes.

We would like to emphasize that our prototype is the first case study of the interplay among network coding, source coding, OFDM and cognitive radio. 

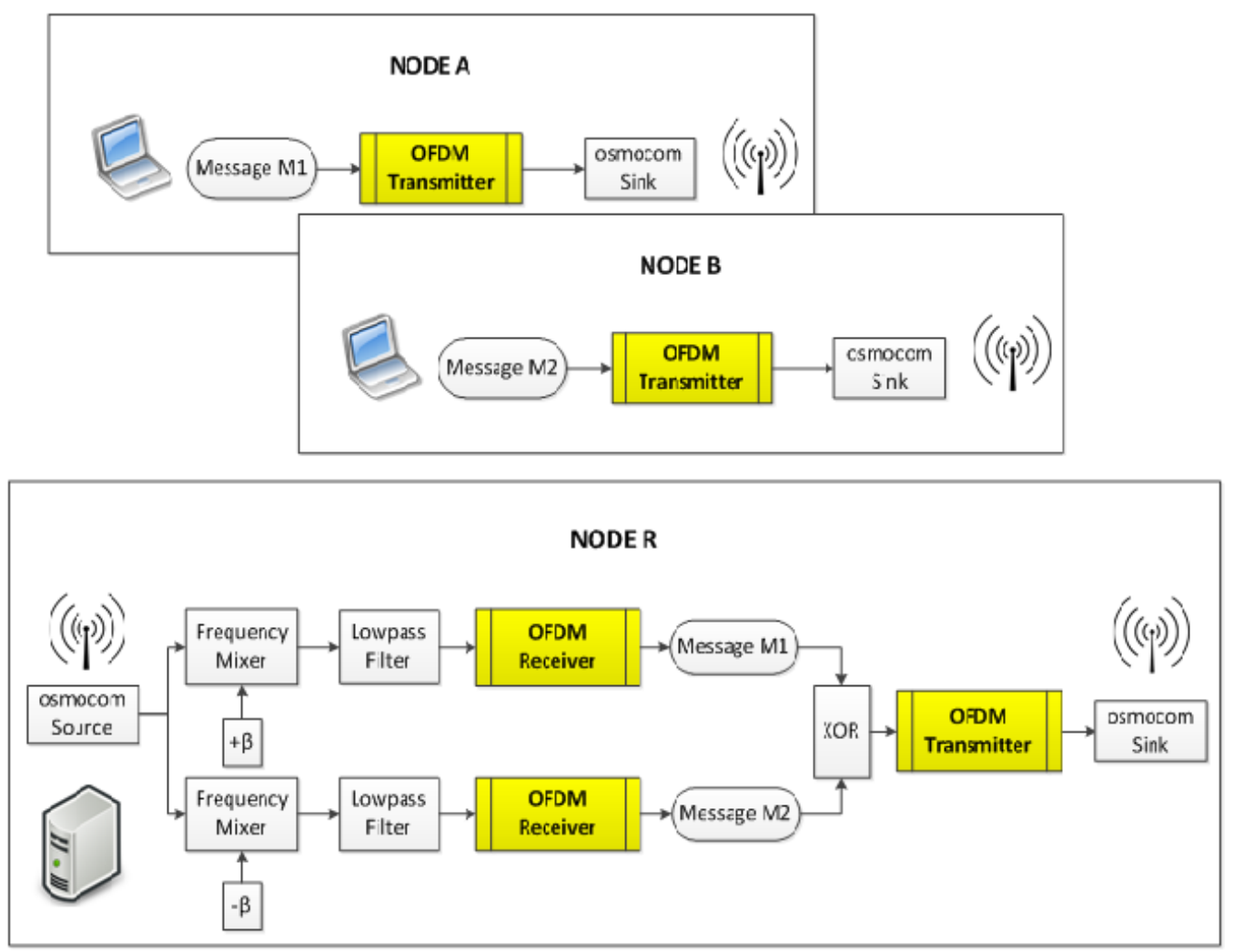

Figure 3. A system block diagram of the TWR model.

In our implementation, we use the version 3.7.6 of GNU Radio running in a dual-core general purpose processor under Ubuntu 14.04 OS. Experiments take place in a $25 \mathrm{~m}^{2}$-area closed laboratory. We place the devices at least $0.5 \mathrm{~m}$ away from each other. We make use of the OFDM tranceiver blocks developed in the gr$s 4 a$ module [13]. Further details about the experimental setup are given in each following section, where we present sequentially the implementation of the three aforementioned scenarios.

\section{Implementation of Network Coding UNDER THE TWR MODEL}

In this section, we focus on an SDR-based real-time implementation of NC under the TWR model.

\subsection{System Model}

We consider the TWR model described in Section 1. To obtain reliable transmission, we need to overcome several challenges, in particular: (i) separation of uplink signals at the relay node, (ii) time and frequency synchronization, and (iii) channel estimation.

Our solutions respectively are: (i) use frequency division multiplexing to distinguish uplink signals at the relay node, (ii) employ OFDM and exploit the preamble part for time and frequency synchronization following the Schmidl-Cox synchronization method, and (iii) insert pilot symbols in an OFDM frame for channel estimation. Next, we will describe these solutions.

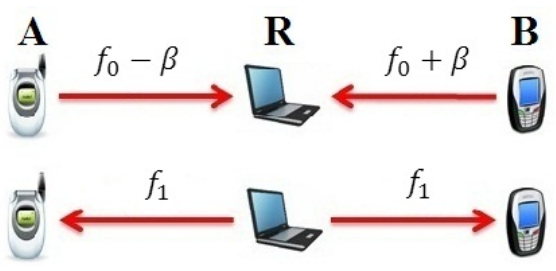

Figure 4. Frequency allocation in the TWR model.

\subsubsection{Separation of Uplink Signals:}

Figure 4 illustrates the frequency allocation in our implementation. Let $f_{0}$ be a (high) carrier frequency. Node $A$ transmits on frequency $f_{0}-\beta$ while node $B$ on frequency $f_{0}+\beta$. Node $R$ transmits on another frequency $f_{1}$. Node $R$ receives on frequency $f_{0}$, but samples with a wide bandwidth enough to completely receive both $f_{0}-\beta$ and $f_{0}+\beta$ so that node $R$ can retrieve signals transmitted from nodes $A$ and $B$ separately.

Figure 3 depicts a signal processing block diagram of the TWR model. In particular, at node $R$, the input signal received at $f_{0}$ goes through two separate branches. In one branch, the input signal is shifted by an amount of $\beta \mathrm{Hz}$ at a frequency mixer, and then the mixed signal is low-pass filtered to retrieve the signal transmitted by node $A$. Similarly, in the other branch, the input signal is shifted by $-\beta \mathrm{Hz}$ at another frequency mixer, and then the mixed signal is low-pass filtered to retrieve the signal transmitted by node $B$. The messages obtained in the two branches after OFDM demodulation are then combined into a new message by the XOR operation. This new message is then modulated and broadcast to $A$ and $B$. 


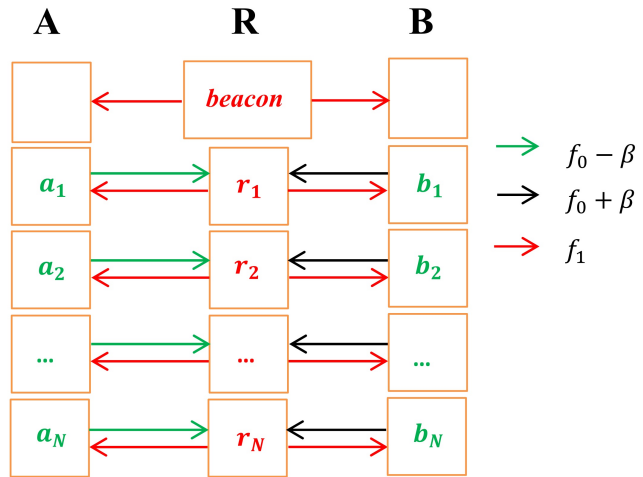

Figure 5. System operating mechanism.

\subsubsection{Communication Protocol:}

Our system works in sessions. The transmission protocol within each session is illustrated in Figure 5. The relay node first broadcasts a beacon message to tell the two end nodes the start of a session. When a session starts, each end node loads $N$ native packets and stores them in a buffer. After that, a checking index $i$ runs from 0 to $N-1$. At each value of $i$, the end node checks whether it has received the corresponding $i$-th XOR-ed packet from the relay or not. If yes, the checking index increases one. If no, the end node transmits the $i$-th native packet and then $i$ is increased by one. If $i=N$, but the end node has not received all $N$ XOR-ed packets yet, it will be returned to zero $(i=0)$. Of course, for the first run of the index $i$ through the buffer, the end node certainly has to send all the loaded native packets. Thus, this protocol allows the end nodes to proceed to the transmission of the next native packet without having to wait for the successful transmission of the corresponding XOR-ed packet from the relay.

At the relay node, whenever it receives a native packet from an end node, it will check whether the corresponding native packet from the other end node is received. If yes, and the XOR-ed packet has not been created yet, the relay node will combine the two corresponding native packets into a XOR-ed packet and store it in a buffer. If no, the received native packet is just stored in a buffer. The XOR-ed packet is broadcast when it is available. A new session starts whenever both end nodes have received all $N$ XOR-ed packets.

\subsubsection{Error Correction Code:}

To improve the communication reliability, we deploy a Hamming $(7,4)$ code with the following generator matrix:

$$
G=\left[\begin{array}{lllllll}
1 & 1 & 1 & 0 & 0 & 0 & 0 \\
1 & 0 & 0 & 1 & 1 & 0 & 0 \\
0 & 1 & 0 & 1 & 0 & 1 & 0 \\
1 & 1 & 0 & 1 & 0 & 0 & 1
\end{array}\right] .
$$

This means that three redundant bits are inserted in the first, second and fourth positions. A 4-bit message is encoded into a 7-bit codeword. At the receiver, the decoder calculates the syndrome metric and estimates the transmitted message. This Hamming code is able to correct one bit error.

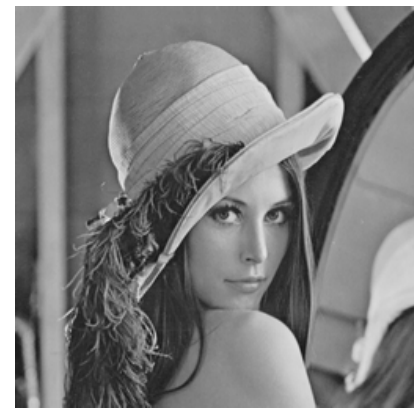

(a) Transmited image at node $A$

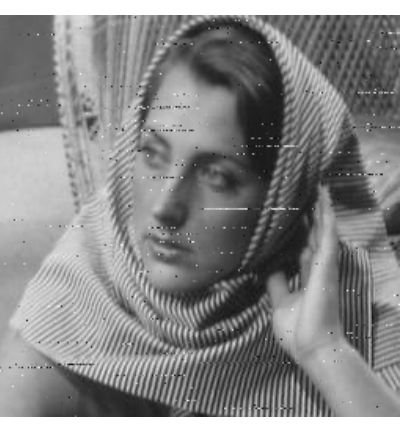

(c) Received image at node $A$ $\mathrm{BER}=0.0128$

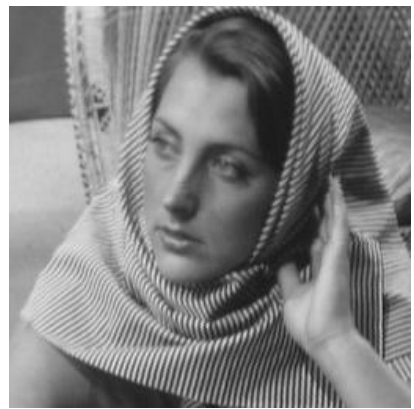

(b) Transmited image at node $B$

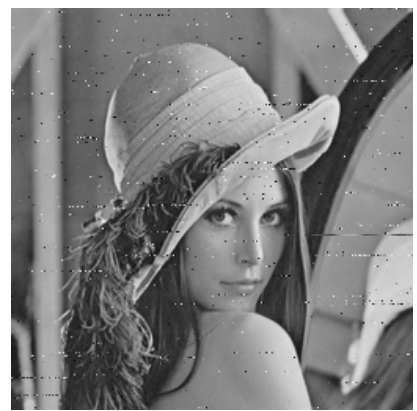

(d) Received image at node $B$, $\mathrm{BER}=0.0122$
Figure 6. Transmitted and received images under the implemented TWR model.

\subsection{SDR Implementation and Results}

As mentioned earlier, we make use of the $g r$-s $4 a$ module [13] for OFDM modulation and demodulation. To make the system work with the operating mechanism described above, we develop some controller blocks for the two end nodes and the relay node. We demonstrate the exchange of two images between the two end nodes. Each end node transmits a $256 \times 256$ image. An instance of transmitted and received images is shown in Figure 6. It is observed that the transmitted images from a node were well retrieved at the other node with a bit error rate (BER) of around $10^{-2}$.

\section{IMPLEMENTATION OF JOINT Source-Network CodING}

Based on the implementation of NC under the TWR model described in Section 3, we extend the implementation to a 4-node network with joint sourcenetwork coding to show the usefulness of $\mathrm{NC}$ for multimedia transmission.

We consider two types of source coding. The first type is layered coding (LC), which is widely used in multimedia source coding. It generates one base layer and some $n$ enhanced layers. The base layer is the most important layer and essential for the data stream to be recovered. Without receiving the base layer, the data stream cannot be recovered since the use of other enhanced layers depends on the content of the base layer. The enhanced layers are to improve the quality of the data stream. However, the first enhanced layer depends on the base layer and each enhanced layer $n+1$ depends on enhanced layer $n$. Thus a certain 


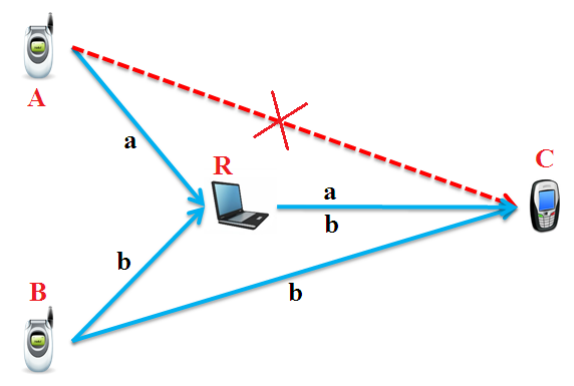

(a) Direct link $A-C$ is lost

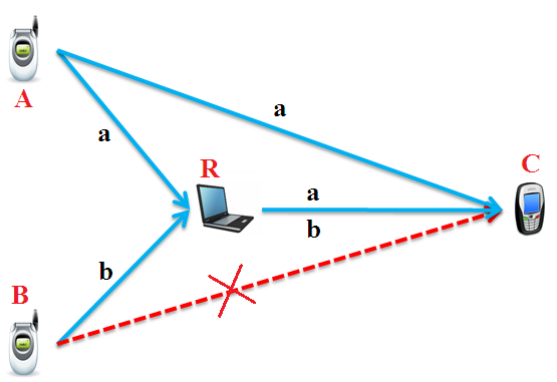

(b) Direct link $B-C$ is lost

Figure 7. 4-node network model with conventional relay.

layer $n$ can only be applied if $n-1$ layers were already applied. Hence, data streams using this layer coding approach can be interrupted whenever the base layer is missing.

The second type of source coding is multipledescription coding (MDC) in which the data stream is divided into $n$ independent sub-streams $(n \geq 2)$, these sub-streams are called descriptions. Thus, MDC is a form of data partitioning. The packets of a description can be sent over different paths. In contrast to LC where the use of layer $n$ depends on layer $n-1$, every received description of MDC at the destination can be used to recover the original data stream. This means that the quality of the decoded stream is proportional to the number of received descriptions. Since any received description can be used for the decoding process, the data stream is rarely interrupted (except for link loss). The loss of some descriptions only results in reduced quality of the decoded stream.

\subsection{System Model}

\subsubsection{A 4-node Network Coding System Model:}

We consider a wireless network model with 4 nodes illustrated in Figure 2(a), in which nodes $A$ and $B$ are two source nodes, node $C$ is the destination, and node $R$ is the relay. Both $A$ and $B$ want to send data to $C$ and both have the direct link to $C$. Node $R$ works as a relaying station with the aim of assisting the data transmission of $A$ and $B$. Node $R$ will relay all of its received packets to node $C$. The presence of node $R$ in the system is to improve the possibility of receiving data packets at $C$ in case a direct link is lost between $A$ and $C$ (link $A-C$ ) or between $B$ and $C$ (link $B-C$ ).

Consider the situation in which the above 4-node network model employs only traditional relaying. Suppose that one of the two direct-links $(A-C$ or $B-C)$ is lost, as

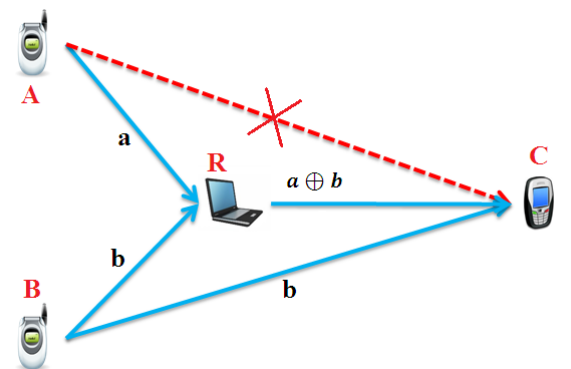

(a) Direct link $A-C$ is lost

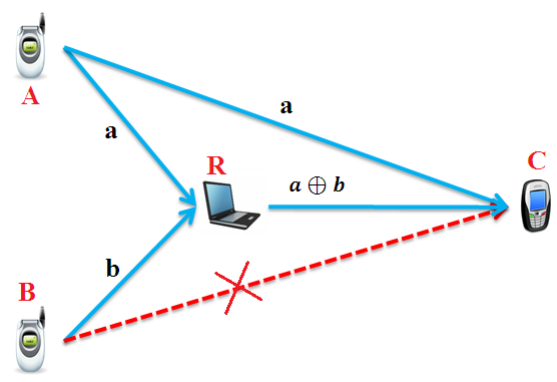

(b) Direct link $B-C$ is lost

Figure 8. 4-node network model with NC.

illustrated in Figure 7. The links $A-R, B-R$, and $R-C$ are supposed to be stable. It can be seen that, thanks to the addition of the relaying station (node $R$ ), $C$ can still receive packets transmitted from $A$ and $B$ even when one of the two direct-links is lost because when node $R$ is active, it relays every packet it receives to $C$.

Now, consider the 4-node network model with $\mathrm{NC}$ as shown in Figure 8. Node $R$ will perform NC on two packets it received ( $a$ and $b$ ) to create a new packet, which is $a \oplus b$, and then forward this new packet to $C$. Suppose that the link between $A$ and $C(A-C)$ is lost as in Figure 8(a). At node $C$, based on the packet $b$ received directly from $B$ and the XOR-ed packet received from $R$, the packet $a$ can be recovered as $a=b \oplus(a \oplus b)$. Similarly, if the link $B-C$ is lost, $b$ can be recovered as $b=a \oplus(a \oplus b)$. Thus, if one of the two direct-links is lost, using NC, node $R$ simply relays the XOR-ed packet to $C$ without knowing which link is lost and still ensures that $C$ can recover both $a$ and $b$. Recall that for the case of using the conventional relaying mechanism, node $R$ has to transmit both $a$ and $b$ since it does not know which direct-link is lost.

4.1.2 A 4-node Joint Source-Network Coding Model:

Now, we combine source coding (at $A$ and $B$ ) with NC (at $R$ ), as shown in Figure 9.

We assume that the direct-link $B-C$ is lost. Each source node ( $A$ or $B$ ) transmits a layer (or a description). Node $R$ performs NC over the two received packets ( $a$ and $b$ ) to create a new coded packet $c$ as follows:

$$
c=a \oplus \beta b,
$$

where $\beta \in\{0,1\}$. We consider the following two cases:

- Case 1: Node $R$ does not have any information about packet $b$, meaning that $b$ is considered as a normal data packet, $\beta$ is set to be 0 or 1 with equal probabilities. 


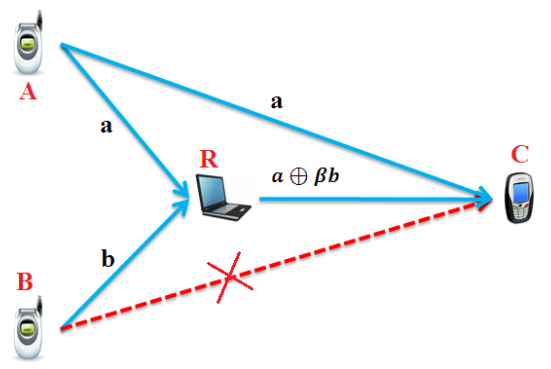

Figure 9. NC with source coding in 4-node network model.

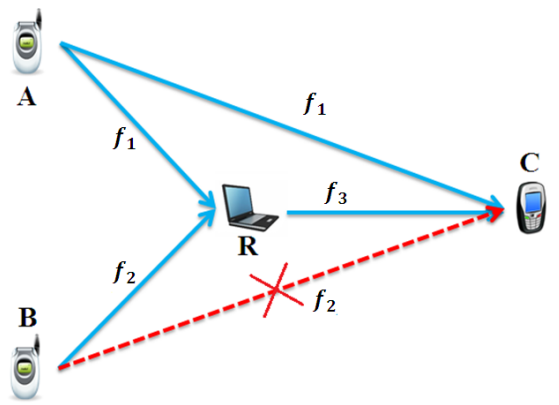

Figure 10. Frequency allocation in 4-node network model.

- Case 2: Node $R$ has information about packet $b$, i.e., $R$ knows if the packet $b$ is of a layer or a description. Essential for the decoding process at $C$, the parameter $\beta$ is set to be 1 . This is to give priority to packets transmitted from $B$.

Figure 10 illustrates the frequency allocation of this 4-node network model. Here we still suppose that the direct-link $B-C$ is lost. The two source nodes $A$ and $B$ transmit on frequencies $f_{1}$ and $f_{2}$, respectively. Node $R$ receives on $f_{1}$ and $f_{2}$, and transmits on $f_{3}$. Since the link $B-C$ is supposed to be lost, node $C$ can only receive signals on $f_{1}$ and $f_{3}$. In addition, node $C$ makes use of a controlling channel $f_{4}$ to transmit control messages to $A$ and $B$. Packets transmitted from $A$ and $B$ will be combined into a XOR-ed packet to be relayed on $f_{3}$. All nodes in the network apply OFDM modulation and demodulation techniques.

This 4-node network also works in sessions. A session starts when node $C$ sends a control message on $f_{4}$ to nodes $A$ and $B$. Therefore, $A$ and $B$ know when to start a session and send their data. Whenever received the control message, end nodes $(A, B)$ will load $N$ packets and then store them in a buffer. After that, end nodes will send $N$ packets continuously until receiving the next control message for the next session.

\subsection{SDR Implementation and Results}

In our implementation, LC/MDC is first performed in Matlab to generate text files containing the layers/descriptions. Then, the controller block of source nodes in GNU radio software loads a text file corresponding to a layer/description and sends it. For simplicity, we implement LC with only two layers (the base layer and one enhanced layer). The data to be coded is a grayscale image. The base layer is generated

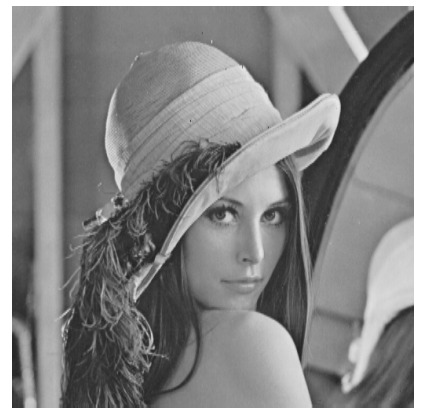

(a) Decoded image using 1 description, BER $=0.1269$

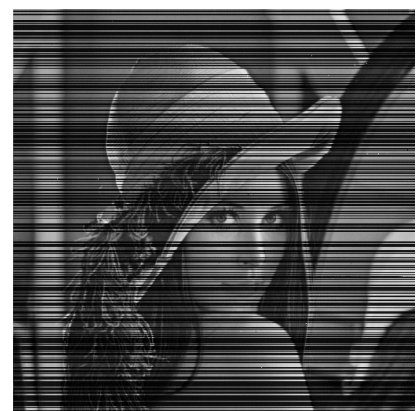

(c) Decoded image without information about source coding, $\mathrm{BER}=0.2673$

Figure 11. Decoded images by LC and MDC.

by filtering the image with a lowpass filter, and the enhanced layer is generated by having the original image subtracted by the base layer. We build a block in GNU Radio for decoding at the destination so that the image can be recovered directly in GNU Radio. Similarly, for MDC, we implement MDC with only two descriptions. Received descriptions at the destination are used to recover the original image. This is done by a block in GNU Radio. For the case of LC, $A$ transmits the enhanced layer and node $B$ transmits the base layer. For the case of MDC, node $A$ transmits one description and node $B$ transmits the other description. Each source node is a commodity PC connected to a bladeRF device. For two nodes $R$ and $C$, each node is a PC connected to two bladeRF devices.

Experimental results are shown in Figure 11. It is clearly seen that the decoded image quality is increased as more descriptions/information about source coding are used in decoding.

\section{IMPLEMENTATION OF VFDM-BASED Cognitive Radio with Network Coding}

Cognitive radio has been attracting a sustained attention for its potential to improve spectral efficiency. Cognitive radio enables the deployment of a two-tier network, composed of a primary tier and a secondary tier. The former is licensed to use a specific spectrum range, whereas the latter accesses this spectrum to carry their transmission without interfering primary transmissions. To avoid cross-tier interference to primary users, secondary users must adapt their transmission 


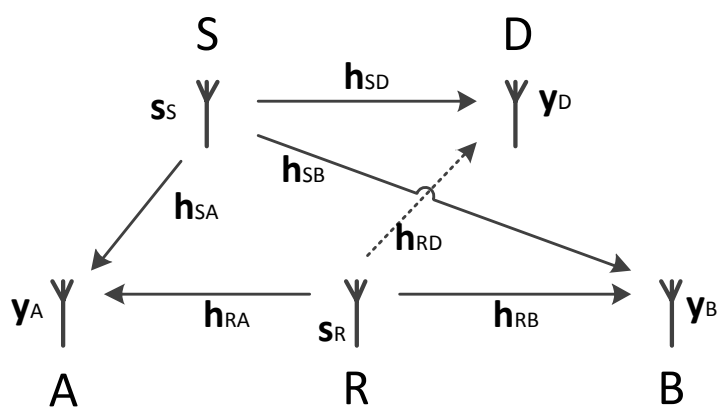

Figure 12. A transmission phase of the cognitive radio network.

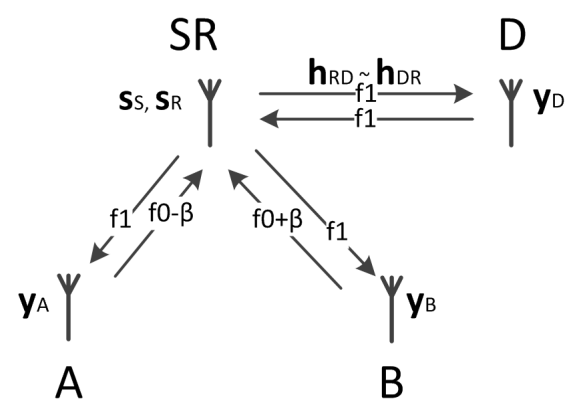

Figure 13. A 4-node hybrid cognitive radio network model. under some constraints in terms of signal power and channel access time. However, these constraints make reliable transmission challenging for secondary users. To guarantee an acceptable reliability for the secondary transmissions, NC, with its potential to improve the network throughput and shorten the transmission time, can be applied at the secondary tier.

Based on the overlay approach, VFDM was proposed in [14] as a technique for cross-tier interference management. With VFDM, a secondary transmitter uses linear precoding to project its signal onto the null space of the interfering channel from the secondary transmitter to the primary receiver. Therefore, the secondary transmitter can transmit over the same band as the primary transmitter but does not cause any interference at the primary receiver.

There have been some results on VFDM implementation in a VFDM standalone transceiver pair [15] or a two transmitter - two receiver scenario, where both transmitters are implemented on the same baseband transceiver, called hybrid transceiver [16]. This hybrid transceiver approach for cognitive network deployment was proposed in [17].

In this section, we present an implementation of a two-tier cognitive network adopting $\mathrm{NC}$ at the secondary tier.

\subsection{System Model}

Consider a 5-node two-tier network scenario, as shown in Figure 2(b), where a primary system composed of a transceiver pair denoted by $S$ (source)/D (destination), shares the spectrum with an opportunistic secondary system which is a TWR network composed of two end nodes $A, B$ and one relay node $R$. The primary system communicates a message $\mathbf{s}_{S}$ over the licensed frequency band, whereas the secondary system access this spectrum to exchange two messages $\mathbf{s}_{A}$ (from $A$ ) and $\mathbf{s}_{B}$ (from $B$ ) between two end nodes via the relay node.

Consider the first phase of this network depicted in Figure 12, where $S$ performs an OFDM transmission towards $D$ with $N$ subcarriers and a cyclic prefix $(C P)$ of size $L$. The total block length is $N+L$. Node $R$ performs a VFDM transmission with the same size of $N+L$.

Let $\mathbf{s}_{S} \in \mathbb{C}^{N}, \mathbf{s}_{R} \in \mathbb{C}^{L}$ be the transmit symbol vector at $S$ and $R$, respectively, $\mathbf{F} \in \mathbb{C}^{N \times N}$ the unitary dis- crete Fourier transform (DFT) matrix with $[\mathbf{F}]_{k+1, l+1}=$ $\frac{1}{\sqrt{N}} e^{-i 2 \pi \frac{k l}{N}}, k, l=[0,1, \ldots, N-1]$, A the CP insertion matrix of size $(N+L) \times N, \mathbf{E} \in \mathbb{C}^{(N+L) \times L}$ the VFDM precoder matrix.

The precoded transmit vector $\mathbf{x}_{S}$ and $\mathbf{x}_{R} \in \mathbb{C}^{N+L}$ at $S$ and $R$, respectively, are defined as follows:

$$
\begin{aligned}
& \mathbf{x}_{S}=\mathbf{A F ^ { - 1 }} \mathbf{s}_{S} \\
& \mathbf{x}_{R}=\mathbf{E} \mathbf{s}_{R} .
\end{aligned}
$$

Let $\mathbf{h}_{M N}=\left[h_{M N, 0}, \ldots, h_{M N, L}\right]$ be the $(L+1)$-tap fading channel vector, modeling the downlink between the transmitter $M$ and the receiver $N$. The convolution of each precoded symbol vector $\mathbf{x}_{M}$ with corresponding channel $\mathbf{h}_{M N}$ can be modeled as a Toeplitz matrix $\mathbf{H}_{M N} \in \mathbb{C}^{(N+L) \times(N+L)}$. The received signal $\mathbf{y}_{D} \in$ $\mathbb{C}^{N}, \mathbf{y}_{A} \in \mathbb{C}^{N+L}$ and $\mathbf{y}_{B} \in \mathbb{C}^{N+L}$ at $D, A$ and $B$, are respectively

$$
\begin{aligned}
& \mathbf{y}_{D}=\mathbf{F B}\left(\mathbf{H}_{S D} \mathbf{x}_{S}+\mathbf{H}_{R D} \mathbf{x}_{R}+\mathbf{n}_{D}\right) \\
& \mathbf{y}_{A}=\mathbf{H}_{S A} \mathbf{x}_{S}+\mathbf{H}_{R A} \mathbf{x}_{R}+\mathbf{n}_{A} \\
& \mathbf{y}_{B}=\mathbf{H}_{S B} \mathbf{x}_{S}+\mathbf{H}_{R B} \mathbf{x}_{R}+\mathbf{n}_{B}
\end{aligned}
$$

where $\mathbf{B}=\left[\mathbf{0}_{N \times L} \mathbf{I}_{N}\right]$ is the CP removal matrix, $\mathbf{n}_{M}$ is the Gaussian noise vector at receiver $M \in\{A, B, D\}$ with covariance matrix $\sigma^{2} \mathbf{I}$.

By analyzing the interference constraint that $R$ must satisfy, $\mathrm{E}$ can be built so that no interference signal component is perceived at $D$ after the $C P$ removal and DFT. Let $\tilde{\mathbf{H}}_{R D}=\mathbf{F B H}_{R D}$. For a zero cross-tier interference, the following condition must be satisfied:

$$
\tilde{\mathbf{H}}_{R D} \mathbf{x}_{R}=\tilde{\mathbf{H}}_{R D} \mathbf{E s}_{R}=\mathbf{0}_{N} \text {. }
$$

This is achieved by a special Vandermonde matrix construction of the linear procoder $\mathbf{E}$, built from the roots of the polynomial $S(z)$ with $L+1$ coefficients of the interference channel $\mathbf{H}_{R D}$ as $S(z)=\sum_{i=0}^{L} h_{R D, i} z^{-1}$.

\subsection{SDR Implementation}

\subsubsection{Baseband design:}

We consider the hybrid transceiver approach [17]. The primary transmitter $S$ and the secondary relay $R$ are implemented on one baseband transceiver. Consequently, the aforementioned 5-node network model is transformed into a 4-node hybrid network model as shown in Figure 13. The hybrid transceiver is denoted as $S R$. In the implementation test-bed, it is a PC connected to two 


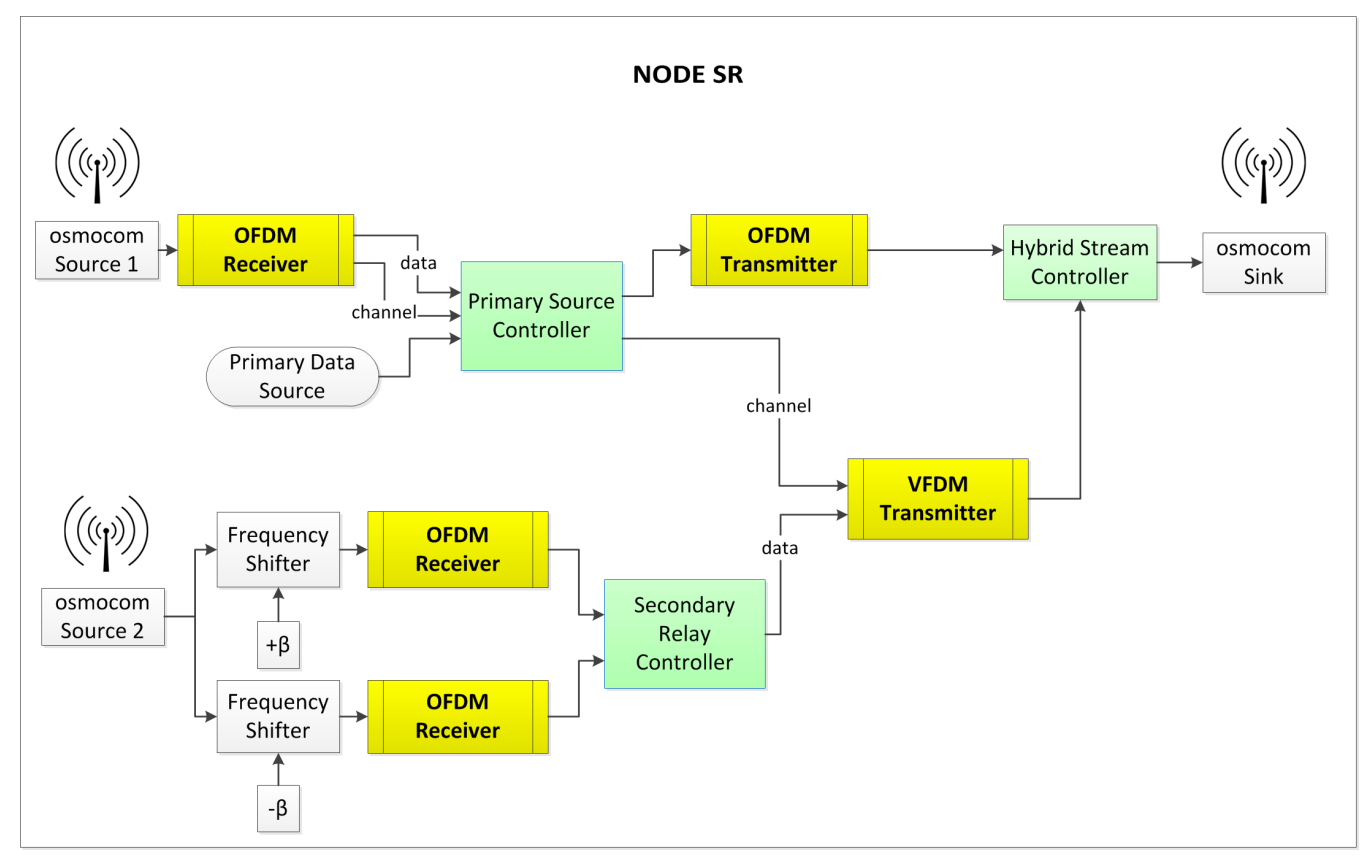

(a) Node $S R$

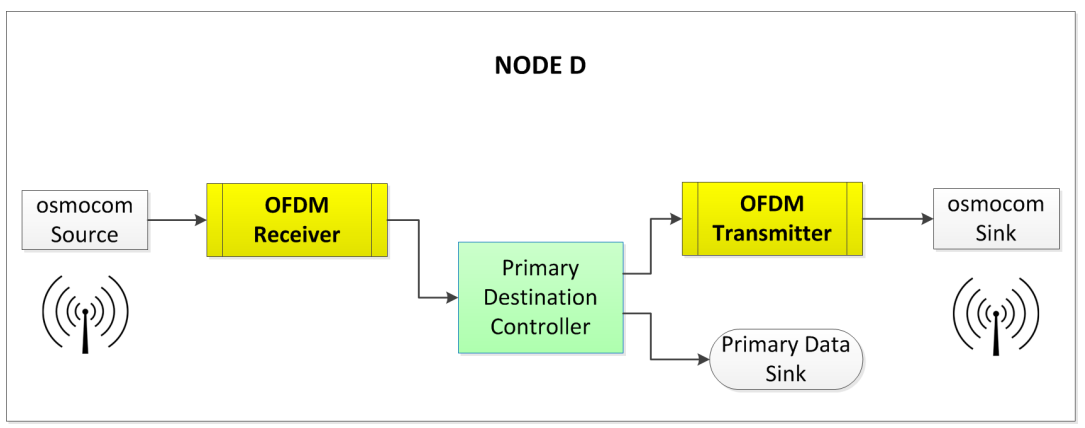

(b) Node $D$

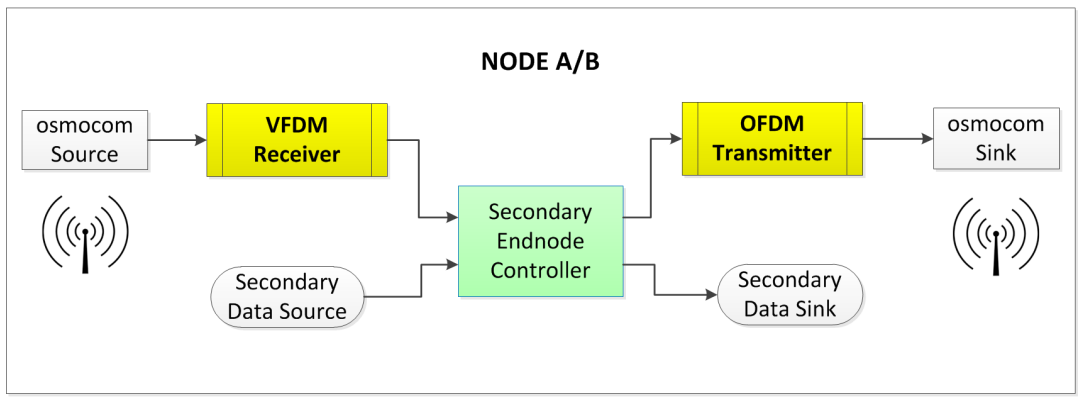

(c) Nodes $A, B$

Figure 14. Signal processing chains of nodes in the considered cognitive radio network.

bladeRF devices. The main motivation for this hybrid approach is that the channel estimate of the primary link can be directly used to build the precoding matrix E for the VFDM transmission.

Each node operates following a specific configuration, depending on its transmitting/receiving mode in uplink/downlink phase and its role in the network. The operating modes and frequency allocated of each node are given in Table I.

An illustration of the signal processing chain in each node is shown in Figure 14. The main algorithms for cognitive transmission strategy are embedded in controller blocks (in green).
Table I

Operating Mode and Frequency Allocated of Nodes for Uplink and Downlink Phases

\begin{tabular}{c|c|c}
\hline Node & Uplink & Downlink \\
\hline$S R$ & OFDM RX, $f_{1}$ & OFDM TX + VFDM TX, $f_{1}$ \\
\hline$D$ & OFDM TX, $f_{1}$ & OFDM RX, $f_{1}$ \\
\hline$A$ & OFDM TX, $f_{0}-\beta$ & VFDM RX, $f_{1}$ \\
\hline$B$ & OFDM TX, $f_{1}+\beta$ & VFDM RX, $f_{1}$ \\
\hline
\end{tabular}

\subsubsection{Communication Protocol:}

We hereafter describe simply the adopted protocol in the order of channel access. The network operates under the channel reciprocity principle in TDD mode. 
In the uplink phase, $D$ sends a message $\mathbf{s}_{S}$ to $S R$ at the center frequency $f_{1}$. This can be either a beacon message to trigger the communication or an ACK message to confirm the successful reception from $S R$ in the previous downlink phase. In both cases, pilot symbols are added to enable the estimation of $\mathbf{H}_{D R}$ at $S R$. At $S R$, thanks to the principle of channel reciprocity, $\mathbf{H}_{D R}$ can be used as $\mathbf{H}_{R D}$ by the VFDM transmitter to construct the precoder E. Meanwhile, in the secondary tier, the end nodes $A$ and $B$ send their message $\mathbf{s}_{A}$ and $\mathbf{s}_{B}$, respectively, to $S R$ at the center frequency $f_{0}+\beta$ and $f_{0}-\beta$, respectively. These signals are carried by frequencies different from that carrying the signal from $D$ and thus cause no interference to the latter. Node $S R$ receives the primary signal from $D$ at center frequency $f_{1}$ and receives the secondary signals at center frequency $f_{0}$ with wide-enough sampling bandwidth to capture both signals from $A$ and $B$.

In the downlink phase, as soon as $S R$ receives the signal from $D$, the downlink phase starts. Both OFDM frame containing the primary data for primary transmission and VFDM frame containing XOR-ed version of received messages from $A$ and $B$ for secondary transmission are generated at $S R$. Two frames are combined for a hybrid frame to be transmitted at $f_{1}$. All the receiving nodes $D, A$ and $B$ receive this frame but demodulate in their custom mode (OFDM or VFDM) to extract their intended data. To be more specific, $D$ demodulates in the OFDM mode while $A$ and $B$ demodulate in the VFDM mode. Afterwards, an ACK message containing a pilot is generated at $D$ to be transmitted back to $S R$ in the next uplink phase in order to acknowledge the successful reception and trigger the next session. The secondary end nodes retrieve the data from the other end node from the received XOR-ed message from $S R$ and its original data.

\subsection{Experimental Results}

In the experiment, we consider carrier frequencies $f_{1}=2.435 \mathrm{GHz}, f_{0}=2.415 \mathrm{GHz}$, and frequency offset $\beta=250 \mathrm{KHz}$. The sampling bandwidth is 1 $\mathrm{MHz}$. We let node $S R$ send a grayscale $(128 \times 128)$ pixel image and focus on the primary transmission result. In Figure 15, we show the transmitted image from $S R$ and decoded image at $D$. The received image at $D$ is at low quality (due to the imperfect channel estimation at $S R$ ) but observable. This first observation shows that the primary system can still communicate its information while allowing the VFDM-based secondary system access its spectrum. Although the number of faulty pixels in Figure 15b is still high, our result serves as a proof for practical feasibility of NC and VFDM in cognitive radio network.

\section{Conclusions}

In this paper, we have presented an implementation of OFDM-based NC in three scenarios, namely, a TWR model, a joint source-network coding for multimedia transmission, and a cognitive radio network adopting

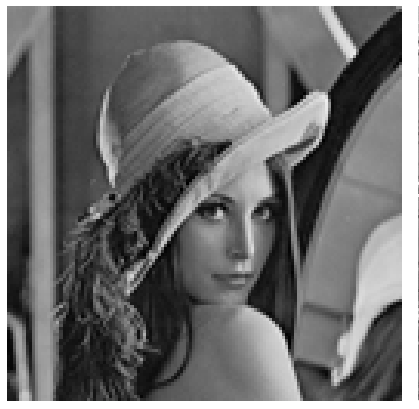

(a) Transmitted image from $S R$

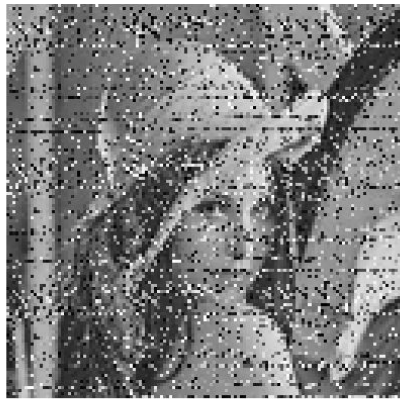

(b) Decoded image at $D$
Figure 15 . The transmitted $(128 \times 128)$-pixel and decoded image in the primary tier of the implemented cognitive radio network.

NC in the secondary tier. Our implementation testbeds operate in real time using the SDR technology. Our results not only complement the theoretical analyses in the literature in showing that NC can improve the network throughput and shorten transmission time, but also confirm the compatibility of NC in practical applications.

\section{ACKNOWLEDGMENT}

This work was partly supported by the Ministry of Science and Technology of Vietnam, under project number 39/2012/HD/NDT, and by the French DIGITEO organization. The authors would like to thank Prof. Muriel Médard from the Department of Electrical Engineering and Computer Science, Massachusetts Institute of Technology, for her invaluable comments to help us improve the perspective of the paper.

\section{REFERENCES}

[1] R. Ahlswede, N. Cai, S.-Y. R. Li, and R. W. Yeung, “Network information flow," IEEE Transactions on Information Theory, vol. 46, no. 4, pp. 1204-1216, 2000.

[2] C. Fragouli, Y. Boudec, J, and J. Widmer, "Network coding: An instant primer," in SIGCOMM Comput. Commun. Rev., vol. 36, no. 1. ACM, Jan. 2006, pp. 63-68.

[3] S. Zhang, S. C. Liew, and P. P. Lam, "Hot topic: Physicallayer network coding," in Proceedings of the 12th Annual international conference on Mobile computing and networking. ACM, 2006, pp. 358-365.

[4] S. C. Liew, S. Zhang, and L. Lu, "Physical-layer network coding: Tutorial, survey, and beyond," Physical Communication, vol. 6, pp. 4-42, 2013.

[5] S. Katti, S. Gollakota, and D. Katabi, "Embracing wireless interference: Analog network coding," in ACM SIGCOMM Computer Communication Review, vol. 37, no. 4. ACM, 2007, pp. 397-408.

[6] [Online] http://gnuradio.org/redmine/projects/gnuradio/wiki.

[7] L. Lu, T. Wang, S. C. Liew, and S. Zhang, "Implementation of physical-layer network coding," Physical Communication, vol. 6, pp. 74-87, 2013.

[8] L. Lu, L. You, Q. Yang, T. Wang, M. Zhang, S. Zhang, and S. C. Liew, "Real-time implementation of physicallayer network coding," in Proceedings of the 2nd Workshop on Software Radio Implementation Forum. ACM, 2013, pp. 71-76.

[9] D. Kramarev, Y. Hong, and E. Viterbo, "Software defined radio implementation of a two-way relay network with 
digital network coding," in Australian Communications Theory Workshop (AusCTW). IEEE, 2014, pp. 120-125.

[10] M. Taghouti, M. Tomoskozi, M. Howeler, D. E. Lucani, F. H. P. Fitzek, A. Bouallegue, and P. Ekler, "Implementation of network coding with recoding for unequalsized and header compressed traffic," in IEEE Wireless Communications and Networking Conference (WCNC), 2019, pp. 1-7.

[11] S. Kafaie, Y. P. Chen, O. A. Dobre, and M. H. Ahmed, "Network coding implementation details: A guidance document," arXiv preprint arXiv:1801.02120, 2018.

[12] [Online] http://nuand.com/.

[13] E. Bastug, "Study of Vandermonde frequency division multiplexing on software defined radio platform," Master of Science, Fatih University, Istanbul, Turkey, May 2012.

[14] L. S. Cardoso, M. Kobayashi, O. Ryan, and M. Debbah, "Vandermonde frequency division multiplexing for cognitive radio," in IEEE 9th Workshop on Signal Processing Advances in Wireless Communications (SPAWC), 2008, pp. 421-425.

[15] M. Maso, L. S. Cardoso, E. Bastug, N. Linh-Trung, M. Debbah, and O. Ozdemir, "On the practical implementation of VFDM-based opportunistic systems: issues and challenges," REV Journal on Electronics and Communications, vol. 2, no. 1-2, pp. 1-18, 2012.

[16] M. Maso, E. Baştuğ, L. S. Cardoso, M. Debbah, and Ö. Özdemir, "Reconfigurable cognitive transceiver for opportunistic networks," EURASIP Journal on Advances in Signal Processing, vol. 2014, no. 1, pp. 1-18, 2014.

[17] L. S. Cardoso, M. Maso, and M. Debbah, "A green approach to femtocells capacity improvement by recycling wasted resources," in IEEE Wireless Communications and Networking Conference (WCNC), 2013, pp. 3817-3822.

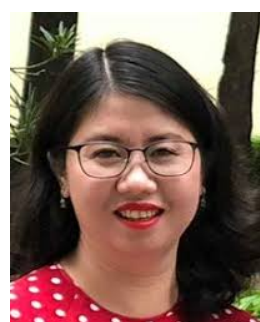

Tran Thi Thuy Quynh was born in 1979. She received the B.Sc., M.Sc. and Ph.D. degrees in Telecommunication Engineering from the University of Engineering and Technology, Vietnam National University, Hanoi, Vietnam in 2001, 2005, and 2016 respectively. Since 2009, she has been on the Faculty of Electronics and Telecommunications, VNU-UET as a researcher. Her research interests include microwave component and antenna design, applying signal processing methods for antenna arrays, and current focusing on implementing of test-beds for networking and cyber-security.

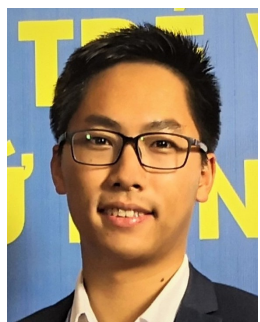

Ngo Khac Hoang received the B.Eng. degree (Hons.) in electronics and telecommunications from University of Engineering and Technology, Vietnam National University, Hanoi, Vietnam, in 2014; and the M.Sc. degree (Hons.) and Ph.D. degree in wireless communications from CentraleSupélec, Paris-Saclay University, France, in 2016 and 2020, respectively. His Ph.D. thesis was also realized at Mathematical and Algorithmic Sciences Laboratory, Huawei Technologies France. Since September 2020, he has been working as a postdoctoral researcher at Communication Systems Group, Department of Electrical Engineering, Chalmers University of Technology, Gothenburg, Sweden. He is also an adjunct researcher at the Advanced Institute of Engineering and Technology of VNU-UET. His research interests include wireless communications and information theory, with an emphasis on MIMO, noncoherent communications, edge computing, massive random access, coded caching, and network coding.

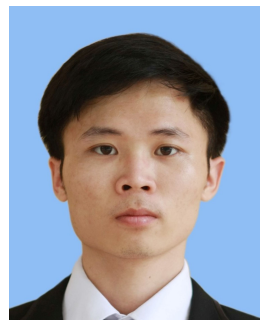

Nguyen Van Ly received the B.Eng. degree in electronics and telecommunications from University of Engineering and Technology (VNUUET), Vietnam National University, Hanoi, Vietnam, in 2014, and the M.Sc. degree in advanced wireless communications systems from CentraleSupélec, Paris-Saclay University, France, in 2016. He is currently pursuing the Ph.D. degree in a joint doctoral program in computational science with San Diego State University and University of California, Irvine, CA, USA, and also an adjunct researcher at the Advanced Institute of Engineering and Technology of VNU-UET. He received a Best Paper Award from the IEEE International Conference on Communications (ICC) in 2020. His research interests include wireless communications, signal processing, and machine learning.

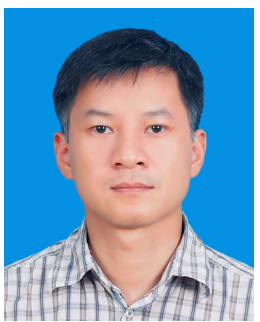

Nguyen Linh Trung obtained his B.Eng. and Ph.D. degrees, both in Electrical Engineering, from Queensland University of Technology, Brisbane, Australia, in 1998 and 2005. Since 2006, he has been on the faculty of the University of Engineering and Technology (UET), Vietnam National University, Hanoi (VNU), where he is currently an associate professor of electronic engineering in the Faculty of Electronics and Telecommunications and director of the Advanced Institute of Engineering and Technology (AVITECH). He is interested in signal processing methods, including time-frequency signal analysis, blind processing, adaptive filtering, compressive sampling, tensor-based signal analysis, graph signal processing, and apply them to wireless communication and networking, biomedical engineering.

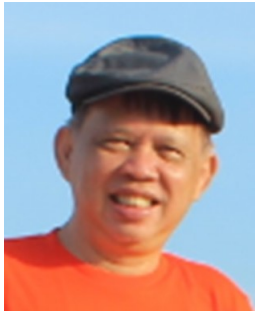

Nguyen Quoc Tuan was born in Hoa Binh Vietnam, in 1953. He received the B. Eng. degree in Radio Physics from University of Hanoi in 1980. He was a faculty member of Hanoi University of Science. Since 1996, he has been on the Faculty of Electronics and Telecommunications, University of Engineering and Technology, Vietnam National University, Hanoi, where he is currently an Associate Professor in Telecommunication Engineering. His research interests include radio physics, communication and networking technologies, including signal processing and system programming.

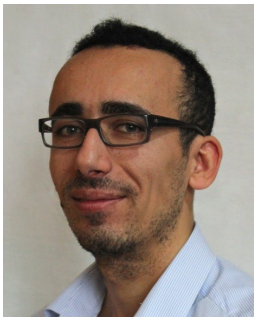

Ejder Bastug is a Member of Technical Staff at Bell Labs since 2018, associated with ENSA lab, coordinating with Sameer Sharma, concentrating on disruptive networking paradigms as part of Future $\mathrm{X}$ vision. Between 2016-2017, he was a postdoctoral researcher at MIT and CentraleSupélec, working with Muriel Médard and Mérouane Debbah respectively. He obtained his Ph.D. at CentraleSupélec in December 2015, focusing on distributed caching methods in small cell networks. He was in the executive or technical committee of flagship conferences, including IEEE WCNC 2014, EuCNC 2015, IEEE BlackSeaCom 2015, IEEE ICC 2017, PIMRC 2019, and WCNC 2021. He is also a regular TPC member of IEEE conferences, and chair of several international workshops. He is currently an associate editor of IEEE Communications Letters. His research interests are in the field of deterministic networking, machine learning and communications. 


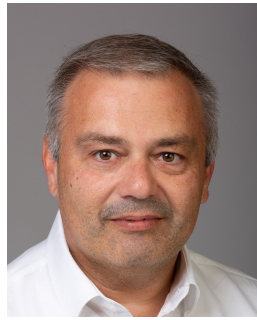

Sylvain Azarian is currently Chief Technical Officer of SDR-Technologies, a French startup working on distributed spectrum sensing and anomaly detections. He funded the company in 2016 while he was project manager to the Director the Electromagnetism and Radar Departement (DEMR) at ONERA, the French Aeronautics, Space and Defense research lab and Director of SONDRA, a joint research laboratory between France and Singapore. From 2009 to 2014 he was Research Engineer at the Alcatel Lucent Chair on Flexible Radio. His research interests include wireless communications, signal processing, radar and cognitive radio.

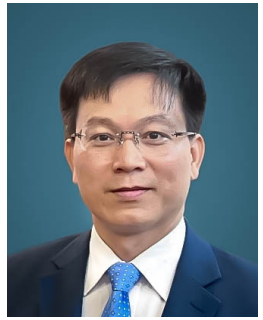

Tran Xuan Nam is currently a professor and head of the research group in advanced wireless communications in Le Quy Don Technical University, Vietnam. He received his Master of Engineering (ME) in Telecommunications Engineering from University of Technology Sydney, Australia in 1998, and Doctor of Engineering in Electronic Engineering from The University of Electro-Communications, Japan in 2003. From November 2003 to March 2006 he was a research associate at the Information and Communication Systems Group, Department of Information and Communication Engineering, The University of Electro- Communications, Tokyo, Japan. Dr. Tran's research interests are in the areas of space-time signal processing for communications such as adaptive antennas, space-time coding, MIMO, spatial modulation and cooperative communications.

Dr. Tran is a recipient of the 2003 IEEE AP-S Japan Chapter Young Engineer Award, and a co-recipient of two best papers from The 2012 International Conference on Advanced Technologies for Communications and The 2014 National Conference on Electronics, Communications and Information Technology. He is a member of IEEE, IEICE and the Radio-Electronics Association of Vietnam (REV).

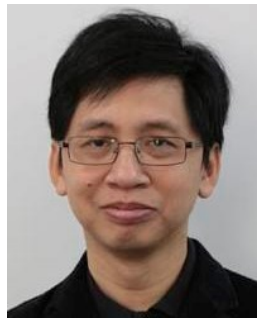

Le Vu Ha is currently Head of the Signals and Systems Laboratory at Faculty of Electronics and Telecommunications, VNU University of Engineering and Technology (VNU UET). He is also leader of the Neurotech research group of the Advanced Institute of Engineering and Technology (AVITECH) at VNU UET. His research and teaching interests include braincomputer interface, machine vision and 3D vision, automatic image and video annotation, super-resolution imaging, bio-medical imaging and signal processing, and multimedia communications.

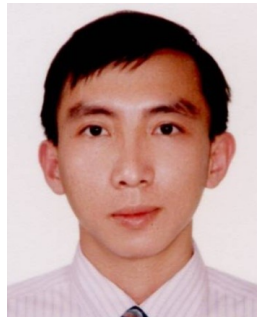

Vo Nguyen Quoc Bao (SMIEEE) is an associate professor of Wireless Communications at Posts and Telecommunications Institute of Technology (PTIT), Vietnam. He is currently serving as the Dean of Faculty of Telecommunications and the Director of the Wireless Communication Laboratory (WCOMM). His research interests include wireless communications and information theory with current emphasis on MIMO systems, cooperative and cognitive communications, physical layer security, and energy harvesting. He is the Technical Editor in Chief of REV Journal on Electronics and Communications. He is also serving as an Associate Editor of EURASIP Journal on Wireless Communications and Networking, an Editor of Transactions on Emerging Telecommunications Technologies (Wiley ETT), and VNU Journal of Computer Science and Communication Engineering. He served as a Technical Program co-chair for ATC $(2013,2014,2018)$, NAFOSTEDNICS (2014, 2015, 2016), REV-ECIT (2015, 2017), ComManTel (2014, 2015), and SigComTel $(2017,2018)$. He is a Member of the Executive Board of the Radio-Electronics Association of Vietnam (REV) and the Electronics Information and Communications Association Ho Chi Minh City (EIC). He is currently serving as vice chair of the Vietnam National Foundation for Science and Technology Development (NAFOSTED) scientific Committee in Information Technology and Computer Science (2017-2019).

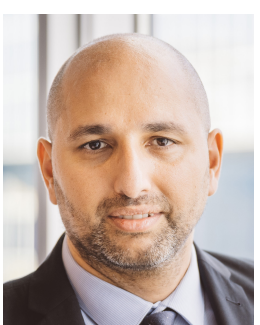

Mérouane Debbah received the M.Sc. and Ph.D. degrees from the Ecole Normale Supérieure Paris-Saclay, France. He was with Motorola Labs, Saclay, France, from 1999 to 2002, and also with the Vienna Research Center for Telecommunications, Vienna, Austria until 2003. From 2003 to 2007, he was an Assistant Professor with the Mobile Communications Department, Institut Eurecom, Sophia Antipolis, France. In 2007, he was appointed Full Professor at CentraleSupelec, Gifsur-Yvette, France. From 2007 to 2014, he was the Director of the Alcatel-Lucent Chair on Flexible Radio. Since 2014, he has been VicePresident of the Huawei France Research Center. He is jointly the director of the Mathematical and Algorithmic Sciences Lab as well as the director of the Lagrange Mathematical and Computing Research Center. He has managed $8 \mathrm{EU}$ projects and more than 24 national and international projects. His research interests lie in fundamental mathematics, algorithms, statistics, information, and communication sciences research.

He is an IEEE Fellow, a WWRF Fellow, and a Membre émérite SEE. He was a recipient of the ERC Grant MORE (Advanced Mathematical Tools for Complex Network Engineering) from 2012 to 2017. He was a recipient of the Mario Boella Award in 2005, the IEEE Glavieux Prize Award in 2011, the Qualcomm Innovation Prize Award in 2012 and the 2019 IEEE Radio Communications Committee Technical Recognition Award. He received more than 20 best paper awards, among which the 2007 IEEE GLOBECOM Best Paper Award, the Wi-Opt 2009 Best Paper Award, the 2010 Newcom++ Best Paper Award, the WUN CogCom Best Paper 2012 and 2013 Award, the 2014 WCNC Best Paper Award, the 2015 ICC Best Paper Award, the 2015 IEEE Communications Society Leonard G. Abraham Prize, the 2015 IEEE Communications Society Fred W. Ellersick Prize, the 2016 IEEE Communications Society Best Tutorial Paper Award, the 2016 European Wireless Best Paper Award, the 2017 Eurasip Best Paper Award, the 2018 IEEE Marconi Prize Paper Award, the 2019 IEEE Communications Society Young Author Best Paper Award and the Valuetools 2007, Valuetools 2008, CrownCom 2009, Valuetools 2012, SAM 2014, and 2017 IEEE Sweden VT-COM-IT Joint Chapter best student paper awards. He is an Associate Editor-in-Chief of the journal Random Matrix: Theory and Applications. He was an Associate Area Editor and Senior Area Editor of the IEEE TRANSACTIONS ON SIGNAL PROCESSING from 2011 to 2013 and from 2013 to 2014, respectively. 


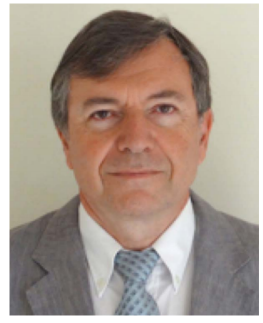

Pierre Duhamel received the Eng. Degree in Electrical Engineering from the National Institute for Applied Sciences (INSA) Rennes, France in 1975, and the Dr. Eng. and the D.Sc degrees from Orsay University, Orsay, France in 1978 and 1986, respectively.

From 1975 to 1980 , he was with ThomsonCSF, Paris, France, where his research interests included circuit theory and signal processing. In 1980, he joined the National Research Center in Telecommunications (CNET), Issy les Moulineaux, France, where his research activities were first concerned with the design of recursive CCD filters. Later, he worked on fast algorithms for computing various signal processing functions (FFT's, convolutions, adaptive filtering, and wavelets. From 1993 to Sept. 2000, he has been professor with ENST (National School of Engineering in Telecommunications), Paris with research activities focused on Signal processing for Communications. He was the head of the Signal and Image processing Department from 1997 to 2000. He is currently with CNRS/LSS (Laboratoire de Signaux et Systemes, Gif sur Yvette, France), where he developed studies in Signal processing for communications and signal/image processing for multimedia applications, including source/protocol/channel coding/decoding. $\mathrm{He}$ is also investigating the connections between communication theory and networking as well as information theory and AI. He has been "directeur de recherches émérite" since March 2019.

He has published more than 100 articles in international journals, more than 300 papers in international conferences, and holds 29 patents. He is a co-author of the book "Joint Source and Channel Decoding: A cross layer perspective with applications in video broadcasting"; which appeared in 2009, Academic Press. He successfully advised or co-advised more than $60 \mathrm{PhD}$ students, and two of them are now fellows of the IEEE.

Dr. Duhamel is a fellow of EURASIP in 2008. He was awarded the "grand prix France Telecom"; by the French Science Academy in 2000. He was a Distinguished lecturer, IEEE, in 1999, and was co-technical chair of ICASSP 06, Toulouse, France and WCNC 2012, Paris, France. A paper on subspace-based methods for blind equalization, which he co-authored, received the "Best paper award" from the IEEE Transactions on Signal Processing in 1998. 